\title{
Geometric Definition and Ideal Aerodynamic Performance of Parabolic Trailing-Edge Flaps
}

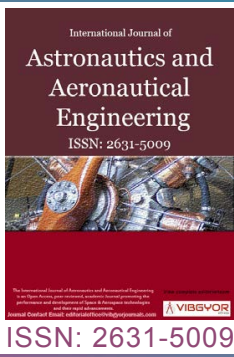

\section{Douglas F Hunsaker ${ }^{*}$, Jackson T Reid ${ }^{2}$ and James $\mathrm{J} \mathrm{Joo}^{3}$}

${ }^{1}$ Assistant Professor, Mechanical and Aerospace Engineering, Utah State University, USA

${ }^{2} \mathrm{PhD}$ Candidate, Mechanical and Aerospace Engineering, Utah State University, USA

${ }^{3}$ Senior Research Mechanical Engineer, U.S. Air Force Research Laboratory, Wright-Patterson Air Force Base, USA

\begin{abstract}
A parabolic trailing-edge flap is defined as a parabolic deflection of the airfoil geometry aft of a hinge point. Whereas a traditional flap deflection causes a discontinuous camber-line slope at the hinge point, a parabolic deflection produces a camber line that is first-order continuous at the hinge point. The geometry manipulation of a parabolic flap is mathematically defined such that it can be applied to any airfoil with a known camber line and thickness distribution. Small-angle and small-camber approximations are used to find analytical predictions for the ideal section flap effectiveness as well as the section pitching moment in comparison to a traditional flap. Results of the parabolic flap are compared to those of a traditional flap producing equivalent lift using thin airfoil theory and the vortex-panel method. It is shown that the ideal section flap effectiveness for a parabolic flap can be $33-50 \%$ higher than that of a traditional flap, depending on the flap-chord fraction. Additionally, a parabolic flap will produce a change in pitching moment $5-50 \%$ greater than that of a traditional flap for a given change in lift. Results may be applied in the design of modern morphing wings, for which complex flap deflections can be produced.
\end{abstract}

\section{Nomenclature}

$A, B, C$ : Constant Coefficients used in Eq. (17); $c$ : Airfoil Chord Length; $c_{f}$ Flap Chord Length; $\widetilde{C}_{L}$ : Airfoil Lift Coefficient; $\widetilde{C}_{L, \alpha}$ : Airfoil Lift Slope; $\widetilde{C}_{m_{c / 4}}$ : Airfoil Pitching-Moment Coefficient about the Quarter Chord; $\widetilde{C}_{m, \delta_{f}}$ : Change in Airfoil PitchingMoment Coefficient about the Quarter Chord with Respect to Flap Deflection for a Traditional Flap, Eq. (53); $\widetilde{C}_{m, \delta_{p}}$ : Change in Airfoil PitchingMoment Coefficient about the Quarter Chord with
Respect to Flap Deflection for a Parabolic Flap, Eq. (52); $l$ : Distance between the Hinge Point and Trailing Edge of an Undeflected Flap, Eq. (15); $R$ : Dimensionless Constant, Eq. (26); $R_{m}$ : Equivalent-Lift Pitching-Moment Ratio, Eq. (61); $R_{\delta}$ : Equivalent-Lift Deflection Ratio, Eq. (55); $R_{\varepsilon}$ : Parabolic-Flap Effectiveness Ratio, Eq. (50); $r$ : Distance between the Hinge Point and a Point on the Camber Line aft of the Hinge, Eq. (8); $t$ : Airfoil Thickness Distribution; $t_{m}$ : Percent Maximum

*Corresponding author: Douglas F Hunsaker, Assistant Professor, Mechanical and Aerospace Engineering, Utah State University, 4130 Old Main Hill, Logan, Utah 84322-4130, USA

Accepted: March 13, 2019; Published: March 15, 2019

Copyright: (C) 2019 Hunsaker DF, et al. This is an open-access article distributed under the terms of the Creative Commons Attribution License, which permits unrestricted use, distribution, and reproduction in any medium, provided the original author and source are credited.

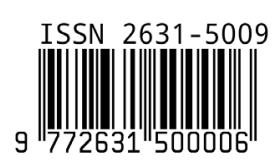

Hunsaker et al. Int J Astronaut Aeronautical Eng 2019, 4:026 
Airfoil Thickness; $x, y$ : Coordinates in the Airfoil Axial and Normal Directions, Respectively; $x_{c}, y_{c}$ : Coordinates of the Airfoil Camber Line Including Flap Deflection; $x_{p} y_{f}$ Coordinates of the Flap Hinge; $x_{p} y_{l}$ : Coordinates along the Airfoil Lower Surface; $x_{m c}: x$-Coordinate of Airfoil Maximum Camber; $x_{o}$ : $x$-Coordinate along the Original Chord Line without Flap Deflection; $x_{p}, y_{p}$ : Coordinates of the Deflected Parabolic Flap Neutral Line, Eqs. (32) and (33); $x_{u^{\prime}}$ $y_{u}$ : Coordinates along the Airfoil Upper Surface; $y_{c 0}: y$-Coordinates of the Airfoil Camber Line with Zero Flap Deflection; $y_{m c}$ : Airfoil Maximum Camber; $y_{n l}: y$-Coordinates of the Undeflected Flap Neutral Line, Eq. (34); $\alpha$ : Airfoil Angle of Attack; $\alpha_{L 0}$ : Airfoil Zero-Lift Angle of Attack; $\gamma$ : Angle between Transformed Flap Neutral Line and the $\xi$-axis, Eq. (22); $\Delta y_{c}$ : Vertical Distance between the Camber Line and the Undeflected Flap Neutral Line, Eq. (35); $\delta_{f}$ : Deflection Angle of a Traditional Flap; $\delta_{p}$ : Deflection Angle of a Parabolic Flap, Eq. (18); $\varepsilon_{i f}$ : Ideal Section Flap Effectiveness of a Traditional Flap, Eq. (49); $\varepsilon_{i p}$ : Ideal Section Flap Effectiveness of a Parabolic Flap, Eq. (48); $\theta$ : Change of Variables for the Chordwise Coordinate, Eq. (44); $\theta_{f}: \theta$ Value at the Flap Hinge Location, $x_{f} ; \xi, \eta$ : Flap Coordinate System Relative to Flap Neutral Line; $\xi_{0}$ : Coordinate along the Undeflected Flap Neutral Line that Corresponds to $\xi_{p}$ along the Deflected Parabolic Flap Neutral Line; $\xi_{p}, \eta_{p}$ : Coordinates of the Deflected Parabolic Flap Neutral Line in the Flap Coordinate System; $\xi_{T E}, \eta_{T E}$ : Coordinates of the Flap Trailing Edge in the Flap Coordinate System; $\phi$ : Local Camber-Line Slope of Undeflected Camber Line, Eq. (12); $\varphi$ : Angle between Flap Neutral Line and $x$-axis, Eq. (16); $\psi$ : Angle of Line Passing through Hinge Point and a Point of Interest on the Flap, Eq. (9)

\section{Introduction}

Modern research for aircraft structures and actuation mechanisms has resulted in the development of new methods for creating changes in camber or initiating flap deflections. For example, the Air Force Research Lab has developed a variablecamber compliant wing (VCCW), capable of changing camber from a NACA 2412 to a NACA 8412 through the use of an embedded actuator [1,2]. A similar technology, currently under development at NASA, is the variable camber compliant trailing edge (VCCTE) [3,4], which can produce variable flap geometries from a series of incremental flap sections. Additionally, shape-memory alloy (SMA) technology can be used for actuation to produce changes to airfoil shape during flight [5]. Activecamber concepts inspired by fish biology are also being investigated [6,7]. The technologies and applications currently under development are quite vast, and we do not attempt an exhaustive list here. Suffice it to say that these technologies allow sophisticated control over airfoil camber, twist, and/or thickness during flight. Advantages of these complex airfoil control methods include reduced RADAR signature, improved aerodynamic efficiency, and passive control. Recent relevant publications include [8-10]. Here we consider one control approach obtained by deflecting the aft portion of an airfoil in a parabolic manner. This will be termed a parabolic flap.

Although the parabolic flap has been studied by other authors $[6,7,11,12]$, a rigorous definition of the resulting airfoil geometry that preserves flap length has not been made. Additionally, a full understanding of the ideal aerodynamic performance of the parabolic flap in comparison to a traditional flap has not been obtained. Because the ideal aerodynamic performance analysis for traditional flap deflections form the foundation of our understanding of traditional flaps, a rigorous definition of the geometry and associated aerodynamic theory is crucial to understanding the benefit of parabolicflap technology. We begin with a brief overview of airfoil geometry including the definitions of a traditional flap, but use a nomenclature conducive to the application of a parabolic-flap deflection.

The surface of an airfoil is defined as the locus of points offset perpendicular to the camber line by one-half the local thickness. The camber line and thickness are often defined as a function of axial position along the airfoil. Traditional airfoil theory is developed using the $x$-coordinate as the axial location along the chord. In order to allow for flap deflections, we will use $x_{o}$ as the coordinate along the original chord line with $x_{c}\left(x_{o}\right)$ and $y_{c}\left(x_{o}\right)$ as the coordinates of the camber line. The thickness at any point along the airfoil is defined as $t\left(x_{0}\right)$. For 
an airfoil without a flap, $x_{c}=x_{o}$ along the length of the entire airfoil. Given camber-line and thickness distributions, the upper and lower coordinates of any airfoil can be computed from

$$
\begin{aligned}
& x_{u}\left(x_{o}\right)=x_{c}\left(x_{o}\right)-\frac{t\left(x_{o}\right)}{2 \sqrt{1+\left(d y_{c} / d x\right)^{2}}} \frac{d y_{c}}{d x} \\
& x_{l}\left(x_{o}\right)=x_{c}\left(x_{o}\right)+\frac{t\left(x_{o}\right)}{2 \sqrt{1+\left(d y_{c} / d x\right)^{2}}} \frac{d y_{c}}{d x} \\
& y_{u}\left(x_{o}\right)=y_{c}\left(x_{o}\right)+\frac{t\left(x_{o}\right)}{2 \sqrt{1+\left(d y_{c} / d x\right)^{2}}} \\
& y_{l}\left(x_{o}\right)=y_{c}\left(x_{o}\right)-\frac{t\left(x_{o}\right)}{2 \sqrt{1+\left(d y_{c} / d x\right)^{2}}}
\end{aligned}
$$

where $d y_{c} / d x$ is the slope of the camber line. For example, a commonly used camber-line distribution is that of the NACA 4-digit series, which is defined as [13]

$$
y_{c 0}\left(x_{o}\right)=\left\{\begin{array}{cc}
y_{m c}\left[2\left(\frac{x_{o}}{x_{m c}}\right)-\left(\frac{x_{o}}{x_{m c}}\right)^{2}\right], & 0 \leq x_{o} \leq x_{m c} \\
y_{m c}\left[2\left(\frac{c-x_{o}}{c-x_{m c}}\right)-\left(\frac{c-x_{o}}{c-x_{m c}}\right)^{2}\right], & x_{m c} \leq x_{o} \leq c
\end{array}\right.
$$

where $x_{m c}$ is the location of maximum camber, $y_{m c}$ is the maximum camber, $c$ is the chord length, and $y_{c 0}$ denotes the $y$-coordinates of the camber line with zero flap deflection. The slope of the camber line for this airfoil series without a flap is

$$
\frac{d y_{c 0}}{d x}\left(x_{o}\right)=\left\{\begin{array}{cl}
2 \frac{y_{m c}}{x_{m c}}\left(1-\frac{x_{o}}{x_{m c}}\right), & 0 \leq x_{o} \leq x_{m c} \\
-\frac{2 y_{m c}}{\left(c-x_{m c}\right)}\left[1-\frac{\left(c-x_{o}\right)}{\left(c-x_{m c}\right)}\right], & x_{m c} \leq x_{o} \leq c
\end{array}\right.
$$

The thickness for the series is defined as

$$
t\left(x_{o}\right)=t_{m}\left[2.969 \sqrt{\frac{x_{o}}{c}}-1.260 \frac{x_{o}}{c}-3.516\left(\frac{x_{o}}{c}\right)^{2}+2.843\left(\frac{x_{o}}{c}\right)^{3}-1.015\left(\frac{x_{o}}{c}\right)^{4}\right]
$$

where $t_{m}$ is the percent maximum thickness. Note that this airfoil definition has a small gap at the trailing edge. An alternate equation for the thickness distribution that does not have a gap at the trailing edge, is $t\left(x_{o}\right)=t_{m}\left[2.980 \sqrt{x_{o} / c}-1.320 x_{o} / c-3.286\left(x_{o} / c\right)^{2}+2.441\left(x_{o} / c\right)^{3}-0.815\left(x_{o} / c\right)^{4}\right]$.

We now consider the geometry of an airfoil with a traditional flap. The flap deflection is created by rotating the locus of points on the original camber line about the point $\left(x_{f}, y_{f}\right)$ by the flap deflection, $\delta_{f}$, with a positive deflection defined as downward. The distance between the hinge point and any point on the 
undeflected camber line aft of the hinge is

$$
r=\sqrt{\left[y_{c 0}\left(x_{o}\right)-y_{f}\right]^{2}+\left(x_{o}-x_{f}\right)^{2}}
$$

The line that passes through the hinge point and the point of interest on the undeflected flap is at an angle relative to the horizontal of

$$
\psi=\tan ^{-1}\left(\frac{y_{c 0}\left(x_{o}\right)-y_{f}}{x_{o}-x_{f}}\right)
$$

The corresponding point on the deflected camber line is found by rotating the original point on the undeflected camber line by the flap deflection, $\delta_{f}$. Applying this rotation to each point aft of the flap hinge gives the airfoil camber-line geometry including deflection

$$
\begin{gathered}
x_{c}=\left\{\begin{array}{cc}
x_{o}, & 0<x_{o}<x_{f} \\
x_{f}+r \cos \left(\delta_{f}-\psi\right), & x_{f}<x_{o}<c
\end{array}\right. \\
y_{c}=\left\{\begin{array}{cc}
y_{c 0}, & 0<x_{o}<x_{f} \\
y_{f}-r \sin \left(\delta_{f}-\psi\right), & x_{f}<x_{o}<c
\end{array}\right.
\end{gathered}
$$

The local camber-line slope for the undeflected flap at any point is $d y_{c 0} / d x$. Defining the local angle of this slope as

$$
\phi \equiv \tan ^{-1}\left(\frac{d y_{c 0}}{d x}\right)
$$

The camber-line slope for the geometry of the deflected flap is

$$
\frac{d y_{c}}{d x}=\left\{\begin{array}{cc}
d y_{c 0} / d x, & 0 \leq x_{o} \leq x_{f} \\
\tan \left(\phi-\delta_{f}\right), & x_{f} \leq x_{o} \leq c
\end{array}\right.
$$

Applying the angle sum identity $\tan \left(\phi-\delta_{f}\right)=\left(\tan \phi-\tan \delta_{f}\right) /\left(1+\tan \phi \tan \delta_{f}\right)$, the camber-line slope at any point on the airfoil with the deflected flap is

$$
\frac{d y_{c}}{d x}=\left\{\begin{array}{cc}
d y_{c 0} / d x, & 0 \leq x_{o} \leq x_{f} \\
\frac{d y_{c 0} / d x-\tan \delta_{f}}{1+\left(d y_{c 0} / d x\right) \tan \delta_{f}}, & x_{f} \leq x_{o} \leq c
\end{array}\right.
$$

The upper and lower surfaces of an airfoil with flap deflection can be found by using Eqs. (10), (11), and (14) in Eqs. (1)-(4). This development can be used to evaluate the geometry of a deflected flap for any airfoil with a given camber-line and thickness distribution. If the vertical position of the flap hinge lies on the camber line, the camber line is continuous at the hinge point. However, this flap deflection introduces a step change in the slope of the camber line at the hinge point. Note that for any positive deflection with a hinge point within the airfoil surface, the lower surface of the airfoil will intersect itself. The same will happen on the upper surface with a negative flap deflection. This geometrical interference can be addressed using various methods, including clipping the geometry or adding a corner radius.

\section{Geometric Definition of a Parabolic Trailing-Edge Flap}

We have characterized a traditional flap as that created by uniform rotation of the camber line aft of the 


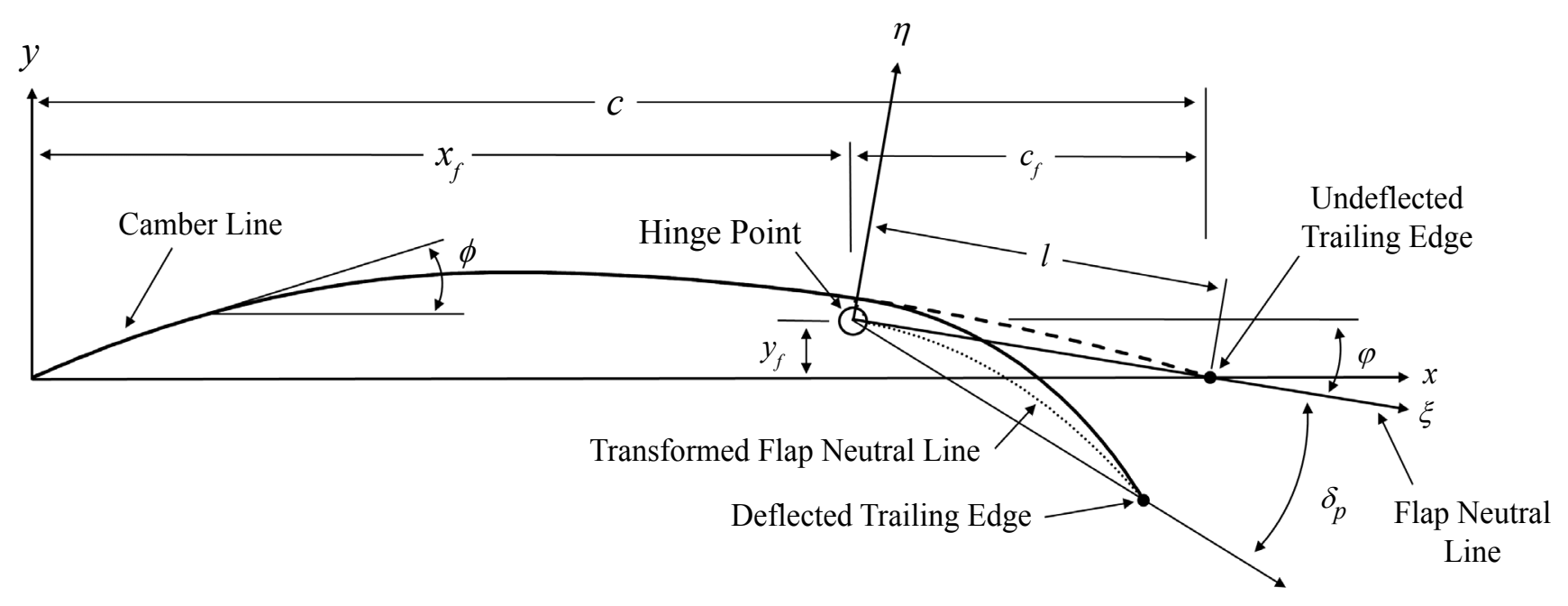

Figure 1: Camber-line geometry for an airfoil section with a parabolic flap at positive deflection.

hinge point. In other words, for a traditional flap deflection, $\delta_{f}$ is constant for all points aft of the hinge point. We now consider the geometric definition of a parabolic trailing-edge flap. At first thought, it may seem most intuitive to define a parabolic trailing-edge deflection as that produced by a linear variation in flap-deflection angle from zero at the hinge point to some finite value at the trailing edge. However, such a deflection produces a geometry with nearly constant curvature along the flap. Because airfoil thickness decreases near the trailing edge, the combination of constant curvature and decreasing thickness produces a strong adverse pressure gradient, which can initiate flow separation. An alternate method that can produce more desirable pressure gradients is that proposed here.

We first define what will be termed the flap neutral line, which is the straight line intersecting the hinge point and the trailing edge. The distance between the hinge point and the trailing edge along this line is

$$
l=\sqrt{y_{f}^{2}+\left(c-x_{f}\right)^{2}}
$$

The flap neutral line sits at an angle to the $x$-axis of $\varphi \equiv-\tan ^{-1}\left(\frac{y_{f}}{c-x_{f}}\right)$

It is convenient to define a flap coordinate system $(\xi, \eta)$ corresponding to the undeflected flap neutral line with the origin at the hinge point, as shown in
Figure 1. This coordinate system is offset from the airfoil coordinate system by $\left(x_{f} y_{f}\right)$, and rotated relative to the airfoil coordinate system by the angle $\varphi$. Here we define a parabolic trailing-edge deflection as that produced by a deflection of the flap neutral line, such that the modified line lies along a parabola in the flap coordinate system, as shown in Figure 1. The position of the camber line relative to the transformed flap neutral line is maintained through the transformation, as well as the airfoil surface relative to the transformed camber line. The dashed line in Figure 1 represents the undeflected camber line, and the thick solid line represents the camber line with flap deflection. For an airfoil with positive camber at a positive flap deflection, the airfoil camber line and upper surface will be lengthened, and the airfoil lower surface will be shortened. However, the length of the flap neutral line will remain the same. Other definitions could be used for defining a parabolic flap deflection, but this definition appears to the authors to be the most geometrically consistent without unnecessarily complicating the geometric definition.

A general form of a parabolic equation, $\eta_{p}(\xi)$, in the flap coordinate system is

$\eta_{p}(\xi)=A \xi^{2}+B \xi+C$

A parabolic deflection of the flap neutral line must satisfy the geometric continuity boundary conditions $\eta_{p}(0)=0$ and $d \eta_{p} / d \xi(0)=0$, which gives $C=0$ and $B=0$. The final coefficient, $A$, is related to the 
flap-deflection angle, $\delta_{p}$, defined here as

$$
\delta_{p} \equiv-\tan ^{-1}\left(\frac{\eta_{T E}}{\xi_{T E}}\right)
$$

where $\left(\xi_{T E}, \eta_{T E}\right)$ is the coordinate of the rotated airfoil trailing edge in the flap coordinate system, and $\delta_{p}$ represents the angle that the trailing edge is rotated about the hinge point, with a positive deflection being downward. Applying the boundary condition in Eq. (18) to Eq. (17) gives

$$
A=-\frac{\tan \delta_{p}}{\xi_{T E}}
$$

Using Eq. (19) in Eq. (17) gives the parabolic relation and first derivative

$$
\begin{aligned}
& \eta_{p}(\xi)=-\frac{\xi^{2}}{\xi_{T E}} \tan \delta_{p} \\
& \frac{d \eta_{p}}{d \xi}=-\frac{2 \xi}{\xi_{T E}} \tan \delta_{p}
\end{aligned}
$$

The angle between the transformed flap neutral line and the $\xi$-axis at any point along the transformed neutral line will be defined here as $\gamma$, i.e.,

$$
\gamma \equiv \tan ^{-1}\left(\frac{d \eta_{p}}{d \xi}\right)=-\tan ^{-1}\left(\frac{2 \xi}{\xi_{T E}} \tan \delta_{p}\right)
$$

In order to complete the geometric definition, we must be able to relate the $\xi$-coordinate of the deflected flap neutral line, $\xi_{p}$, to the $\xi$-coordinate along the original undeflected flap neutral line, $\xi_{0}$. Because the neutral line does not change length during deflection, any coordinate along the original undeflected neutral line is equal to the corresponding length along the parabolic curve of the deflected neutral line. The undeflected $\xi$ -coordinate, $\xi_{0}$, can be found by integrating along the parabolic curve of the deflected neutral line

$$
\xi_{o}=\int_{\xi=0}^{\xi_{p}} \sqrt{1+\left(\frac{d \eta_{p}}{d \xi}\right)^{2}} d \xi
$$

Using Eq. (21) in Eq. (23) and integrating gives the relation between $\xi_{0}$ and $\xi_{p}$ for any point on the flap

$$
\xi_{o}=\frac{\xi_{p}}{2} \sqrt{4 \frac{\xi_{p}^{2}}{\xi_{T E}^{2}} \tan ^{2} \delta_{p}+1}+\frac{\xi_{T E}}{4 \tan \delta_{p}} \sinh ^{-1}\left(2 \frac{\xi_{p}}{\xi_{T E}} \tan \delta_{p}\right)
$$

At the trailing edge, $\xi_{0}=l$ and Eq. (24) can be used to evaluate the $\xi$-coordinate of the deflected trailing edge,

$$
\xi_{T E}=\frac{2 l}{R}
$$

where $R$ is a dimensionless constant that depends on the flap deflection angle

$$
R \equiv \sqrt{4 \tan ^{2} \delta_{p}+1}+\frac{\sinh ^{-1}\left(2 \tan \delta_{p}\right)}{2 \tan \delta_{p}}
$$


The $\eta$-coordinate of the deflected trailing edge can be found by using Eq. (25) in Eq. (20)

$$
\eta_{T E}=-\frac{2 l}{R} \tan \delta_{p}
$$

Using Eq. (25) in Eq. (24) gives $\xi_{0}$ as a function of $\xi_{p}$ and the flap angle

$$
\xi_{o}=\frac{\xi_{p}}{2} \sqrt{\frac{\xi_{p}^{2}}{l^{2}} R^{2} \tan ^{2} \delta_{p}+1}+\frac{l}{2 R \tan \delta_{p}} \sinh ^{-1}\left(\frac{\xi_{p}}{l} R \tan \delta_{p}\right)
$$

For $\delta_{p}=0$, Eqs. (26) and (28) are indeterminate. Thus, in the limit as $\delta_{p} \rightarrow 0$, these equations should be replaced with the leading-order solution from the Taylor series expansion

$$
\begin{aligned}
& R=1+\sqrt{4 \delta_{p}^{2}+1} \\
& \xi_{o}=\frac{\xi_{p}}{2}\left(1+\sqrt{\frac{\xi_{p}^{2}}{l^{2}} R^{2} \delta_{p}^{2}+1}\right)
\end{aligned}
$$

For double-precision computations, roundoff error becomes noticeable for $\delta_{p}<0.0006$ deg. Using Eq. (29) instead of Eq. (26) for $\delta_{\mathrm{p}}<=0.1 \mathrm{deg}$ introduces errors for $R$ on the order of $1.0 \times 10^{-4}$ percent. For $\delta_{\mathrm{p}}<=0.01$ deg, Eq. (29) produces errors on the order of $1.0 \times 10^{-6}$ percent.

With the relations described above, the geometry of the parabolic flap can be computed as follows: given a chord length, $c$, airfoil camber distribution, $y_{c 0}$, airfoil thickness distribution, $t\left(x_{o}\right)$, location of the flap hinge point, $\left(x_{f}, y_{f}\right)$, and flap angle, $\delta_{p}$, Eqs. (15) and (16) can be used to define the flap neutral line. Equation (26) is then used to compute the constant, $R$, which is used in Eqs. (25) and (27) to compute $\xi_{T E}$ and $\eta_{T E}$. Given an axial coordinate aft of the hinge point that lies along the airfoil chord line with zero flap deflection, $x_{o}$, the point on the flap neutral line that corresponds to this same axial location is

$$
\xi_{o}=\frac{\left(x_{o}-x_{f}\right)}{\left(c-x_{f}\right)} l
$$

Equation (28) is then used to solve for the corresponding $\xi_{p}$ value using an iterative solver such as Newton's method. An initial guess of $\xi_{p}=\xi_{T E} \xi_{o} / l$ yields good results. Equations (20) and (21) are used to evaluate the parabolic function and its first derivative at each value of $\xi_{p}$. The coordinates of the deflected parabolic flap neutral line in the flap coordinate system, $\left(\xi_{p}, \eta_{p}\right)$, can be transformed to the airfoil coordinate system $\left(x_{p}, y_{p}\right)$ through the transformation

$$
\begin{aligned}
& x_{p}=x_{f}+\xi_{p} \cos \varphi-\eta_{p} \sin \varphi \\
& y_{p}=y_{f}+\xi_{p} \sin \varphi+\eta_{p} \cos \varphi
\end{aligned}
$$

From the definition of the flap neutral line and Eq. (31), the $y$-coordinate of any point along the undeflected flap neutral line can be found from

$$
y_{n l}=y_{f}\left(1-\frac{\xi_{o}}{l}\right)=y_{f}\left[1-\frac{\left(x_{o}-x_{f}\right)}{\left(c-x_{f}\right)}\right]
$$

The vertical distance between the camber line and the undeflected flap neutral line at any point, $x_{o}$, is

$$
\Delta y_{c}\left(x_{o}\right) \equiv y_{c 0}\left(x_{o}\right)-y_{n l}\left(x_{o}\right)
$$




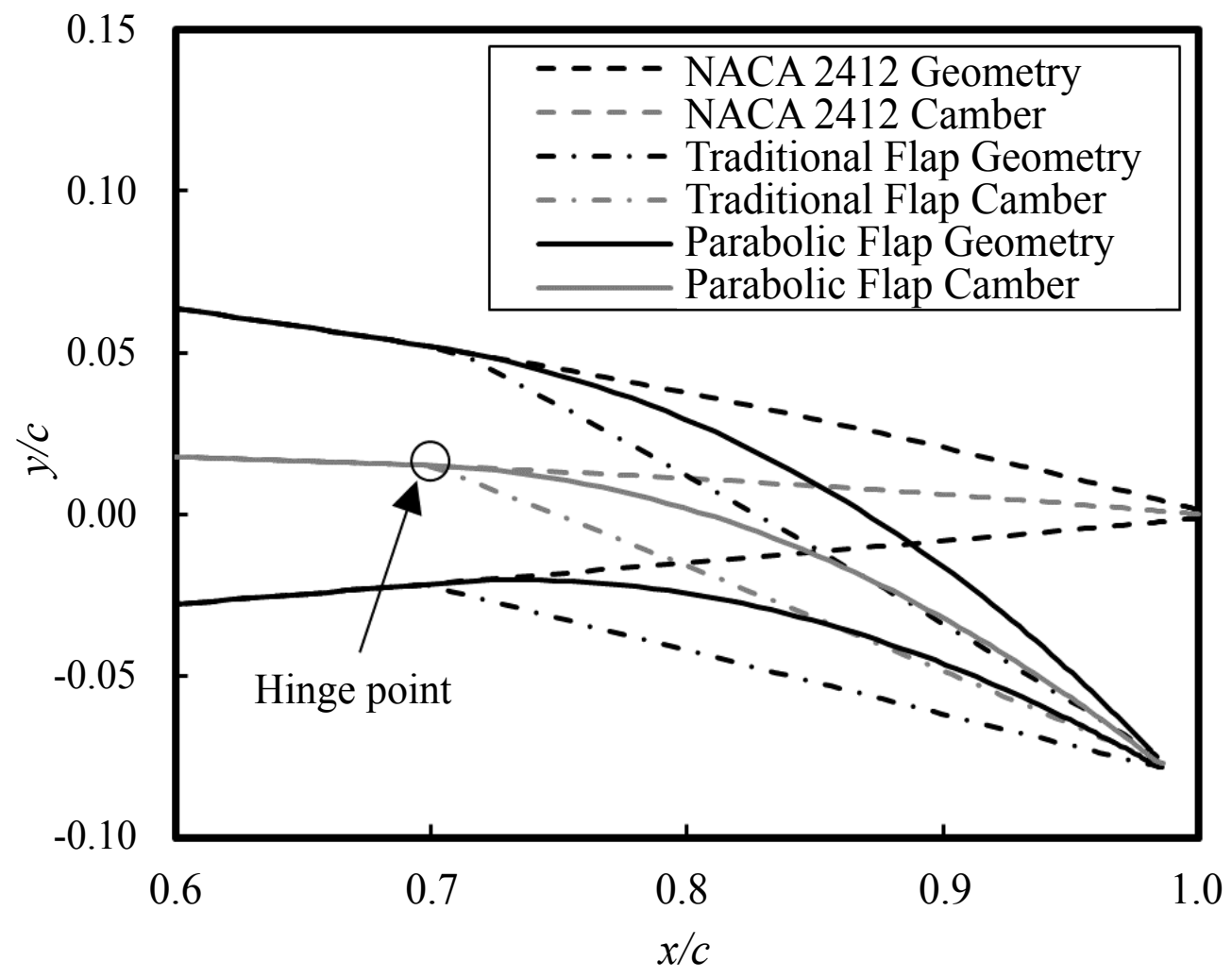

Figure 2: Airfoil geometry and camber line for the NACA 2412 airfoil without flap deflection, with a traditional flap deflection, and with a parabolic flap deflection with $x_{f} / c=0.7$ and $\delta_{f}=\delta_{p}=15 \mathrm{deg}$.

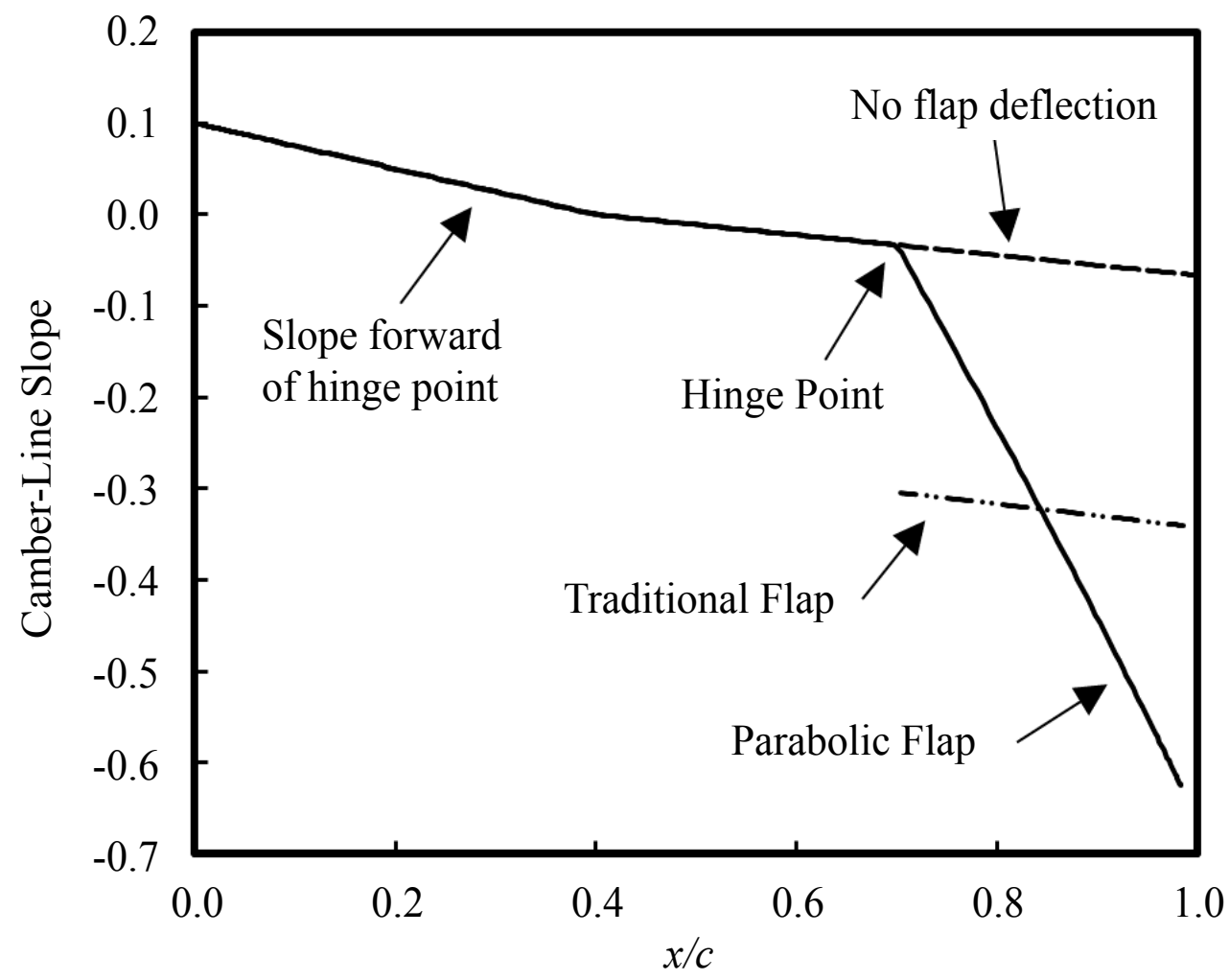

Figure 3: Camber-line slope of the NACA 2412 airfoil without flap deflection, with a traditional flap deflection, and with a parabolic flap deflection with $x_{f} / c=0.7$ and $\delta_{f}=\delta_{p}=15 \mathrm{deg}$. 
The transformed camber line at the point of interest is found by an offset to the transformed flap neutral line, rotated by the change in local angle of the flap neutral line, $\gamma$. Using Eq. (22) gives

$$
\begin{gathered}
x_{c}=\left\{\begin{array}{cc}
x_{o}, & 0<x_{o}<x_{f} \\
x_{p}+\Delta y_{c} \sin \left[\tan ^{-1}\left(\frac{2 \xi_{p}}{\xi_{T E}} \tan \delta_{p}\right)\right], & x_{f}<x_{o}<c
\end{array}\right. \\
y_{c}=\left\{\begin{array}{cc}
y_{c 0}, & 0<x_{o}<x_{f} \\
y_{p}+\Delta y_{c} \cos \left[\tan ^{-1}\left(\frac{2 \xi_{p}}{\xi_{T E}} \tan \delta_{p}\right)\right], & x_{f}<x_{o}<c
\end{array}\right.
\end{gathered}
$$

The slope of the camber line including flap deflection can be found by adding the original camber-line slope given in Eq. (12) to the slope of the transformed flap neutral line, given in Eq. (22), at any point of interest. This gives

$$
\frac{d y_{c}}{d x}=\left\{\begin{array}{cc}
d y_{c 0} / d x, & 0 \leq x_{o} \leq x_{f} \\
\tan (\phi+\gamma), & x_{f} \leq x_{o} \leq c
\end{array}\right.
$$

Using Eqs. (12) and (22) in Eq. (38) and applying the angle sum identity $\tan (\phi+\gamma)=(\tan \phi+\tan \gamma) /(1-\tan \phi \tan \gamma)$, the camber-line slope at any point on the airfoil with the parabolic flap is

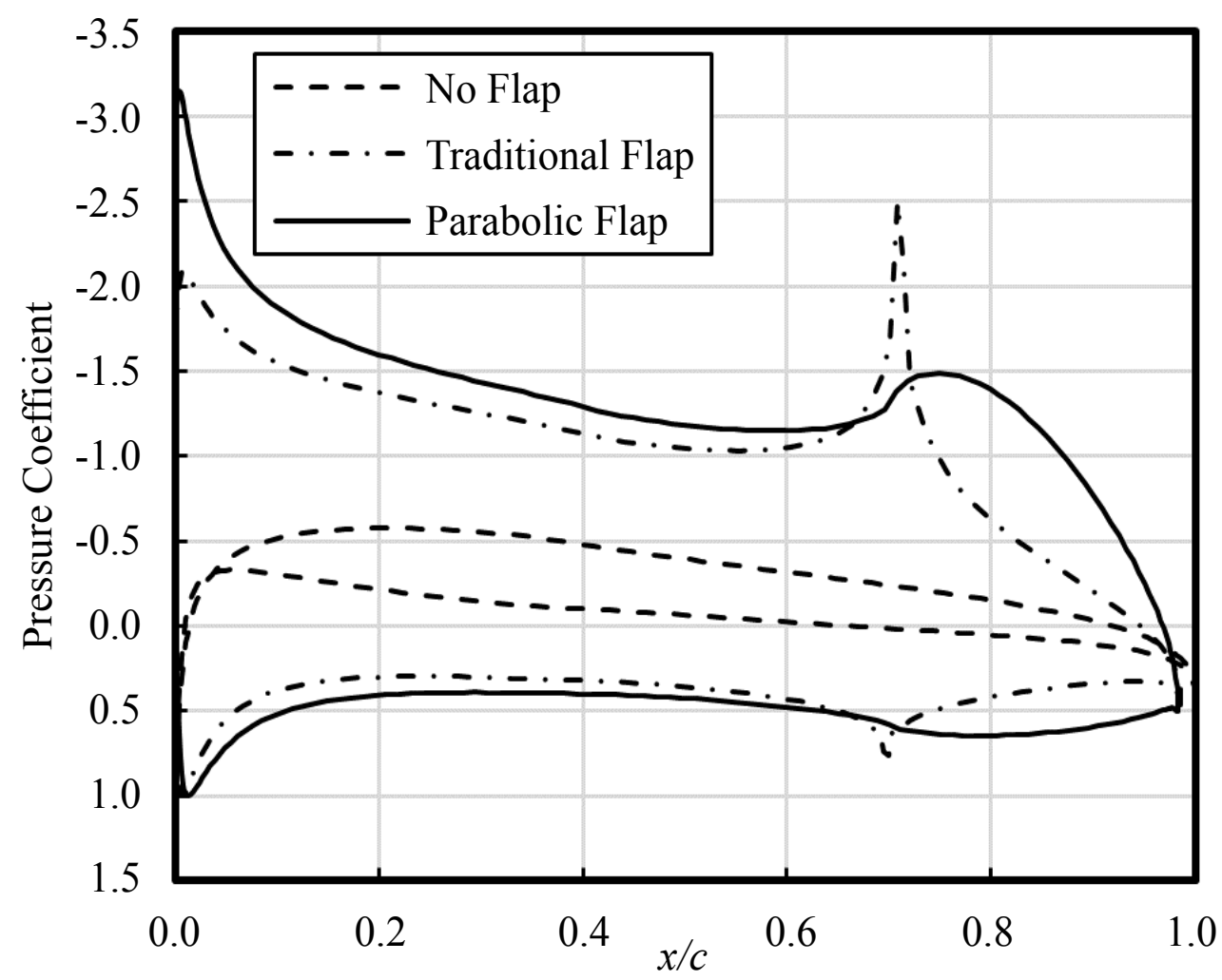

Figure 4: Pressure coefficient along upper and lower surfaces of the NACA 2412 airfoil without flap deflection, with a traditional flap deflection, and with a parabolic flap deflection with $x_{f} / c=0.7, \delta_{f}=\delta_{p}=15 \mathrm{deg}$, and an angle of attack of zero. 


$$
\frac{d y_{c}}{d x}=\left\{\begin{array}{cc}
d y_{c 0} / d x, & 0 \leq x_{o} \leq x_{f} \\
\frac{d y_{c 0} / d x-2 \xi_{p} \tan \delta_{p} / \xi_{T E}}{1+2 \xi_{p}\left(\tan \delta_{p} / \xi_{T E}\right)\left(d y_{c 0} / d x\right)}, & x_{f} \leq x_{o} \leq c
\end{array}\right.
$$

Equations (36), (37), and (39) can then be used in Eqs. (1)-(4) to evaluate the airfoil surface geometry.

Figure 2 shows the surface geometry and camber line of a NACA 2412 airfoil without a flap, with a traditional flap deflection of $\delta_{f}=15 \mathrm{deg}$, and with a parabolic flap deflection of $\delta_{p}=15 \mathrm{deg}$. For each case, the flap hinge location was set to $x_{f} / c=0.7$ and $y_{f} / c=y_{c 0}\left(x_{f}\right) / c=0.015$. Figure 3 shows the associated camber-line slopes, and Figure 4 shows the associated pressure coefficient along the upper and lower surfaces for each case at an angle of attack of zero. Note from Figure 3 that the camber-line slope for the traditional flap has a discontinuity at the hinge point, whereas the camber-line slope of the parabolic flap is continuous across the entire airfoil. The discontinuity in camber-line slope characteristic of traditional flap deflections causes a pressure spike at the hinge point, which can be seen in Figure 4. This large pressure gradient can induce flow separation at or near the hinge point. The parabolic flap does not exhibit a pressure spike at the hinge point, but does have a large adverse pressure gradient near the airfoil trailing edge, which can induce flow separation near the flap trailing edge.

The method outlined in this section can be used to define the geometry of any airfoil with a parabolic flap. This has been demonstrated here using the NACA 4-digit airfoil series, but can be applied to any arbitrary airfoil, provided that the airfoil camber line and thickness are known as a function of axial coordinate. This process preserves the length along the outer surface of symmetric airfoils. For airfoils with non-zero camber, changes to the outer-surface length are extremely small. For example, the surface length of a NACA 8412 airfoil with $x_{f} / c=0.7$ and $\delta_{p}=50$ deg differs from the undeflected surface length by about $0.3 \%$.

\section{Ideal Aerodynamic Performance}

We now consider the ideal aerodynamic performance of a parabolic trailing-edge flap compared to that of a traditional flap. We will approach this through the use of thin airfoil theory, and will include vortex-panel solutions to demonstrate thickness and camber effects. Thin airfoil theory was developed by Max Munk, who published the theory in 1922 as a NACA report [14]. Versions of his theory were soon published with minor modifications by Birnhaum [15] and Glauert [16-18]. Many of the early NACA airfoils were developed using this theory [19], and summaries of the theory can be found in many aerodynamics textbooks [20-26]. Glauert was particularly instrumental in extending the original theory to include the effects of flaps [18]. Glauert's extension to the original theory has been summarized and further discussed by Abbott and von Doenhoff [27] and Phillips [28].

Thin airfoil theory applies the approximations of thin airfoils at small angles of attack, small camber, and small flap deflections to obtain predictions for the lift and quarter-chord pitching-moment coefficients. This theory gives

$$
\begin{gathered}
\tilde{C}_{L}=\tilde{C}_{L, \alpha}\left(\alpha-\alpha_{L 0}\right) \\
\tilde{C}_{m_{c / 4}}=\frac{1}{2} \int_{\theta=0}^{\pi} \frac{d y_{c}}{d x}[\cos (2 \theta)-\cos \theta] d \theta
\end{gathered}
$$

where the lift slope and zero-lift angle of attack are

$$
\widetilde{C}_{L, \alpha}=2 \pi
$$


$\alpha_{L 0}=\frac{1}{\pi} \int_{\theta=0}^{\pi} \frac{d y_{c}}{d x}(1-\cos \theta) d \theta$

and the airfoil $x$-coordinate is related to $\theta$ through the change of variables

$x(\theta)=\frac{c}{2}(1-\cos \theta), \quad \theta=\cos ^{-1}(1-2 x / c)$

Within the limits of thin airfoil theory, the lift slope is independent of the camber line, as can be seen in Eq. (42). Thus, within the limits of this theory, the camber line only affects the lift through its effect on the zero-lift angle of attack, as can be seen from Eq. (43). Additionally, the camber line affects the airfoil pitching moment as can be seen in Eq. (41).

\section{Ideal section flap effectiveness}

Thin airfoil theory can be used to estimate the ideal section flap effectiveness, which is defined as the negative of the change in zero-lift angle of attack with respect to flap deflection for small deflection angles [27,28]. In order to obtain a closed-form approximation for the ideal section flap effectiveness, we will retain the small-camber, small-angle, and small-deflection approximations used in thin airfoil theory. From Eqs. (29), (30), and (25) we obtain the small deflection-angle approximations $\tan \delta_{p} \approx \delta_{p}, \quad R \approx 2, \xi_{p} \approx \xi_{o}, \xi_{T E} \approx l, x_{o} \approx x$, and $\left(2 \xi_{o} \delta_{p} / l\right)\left(d y_{c 0} / d x\right)<<$. Using these small-angle approximations and applying Eq. (31), the camberline slope given in Eq. (39) for a parabolic flap can be written as

$\frac{d y_{c}}{d x}=\frac{d y_{c 0}}{d x}+\left\{\begin{array}{cc}0, & 0 \leq x \leq x_{f} \\ -2 \frac{\left(x-x_{f}\right)}{\left(c-x_{f}\right)} \delta_{p}, & x_{f} \leq x \leq c\end{array}\right.$

This is the same small-angle camber-line slope used by Sanders, Eastep, and Forster [11]. Using Eq. (45) in Eq. (43) with the change of variables given in Eqs. (44) gives

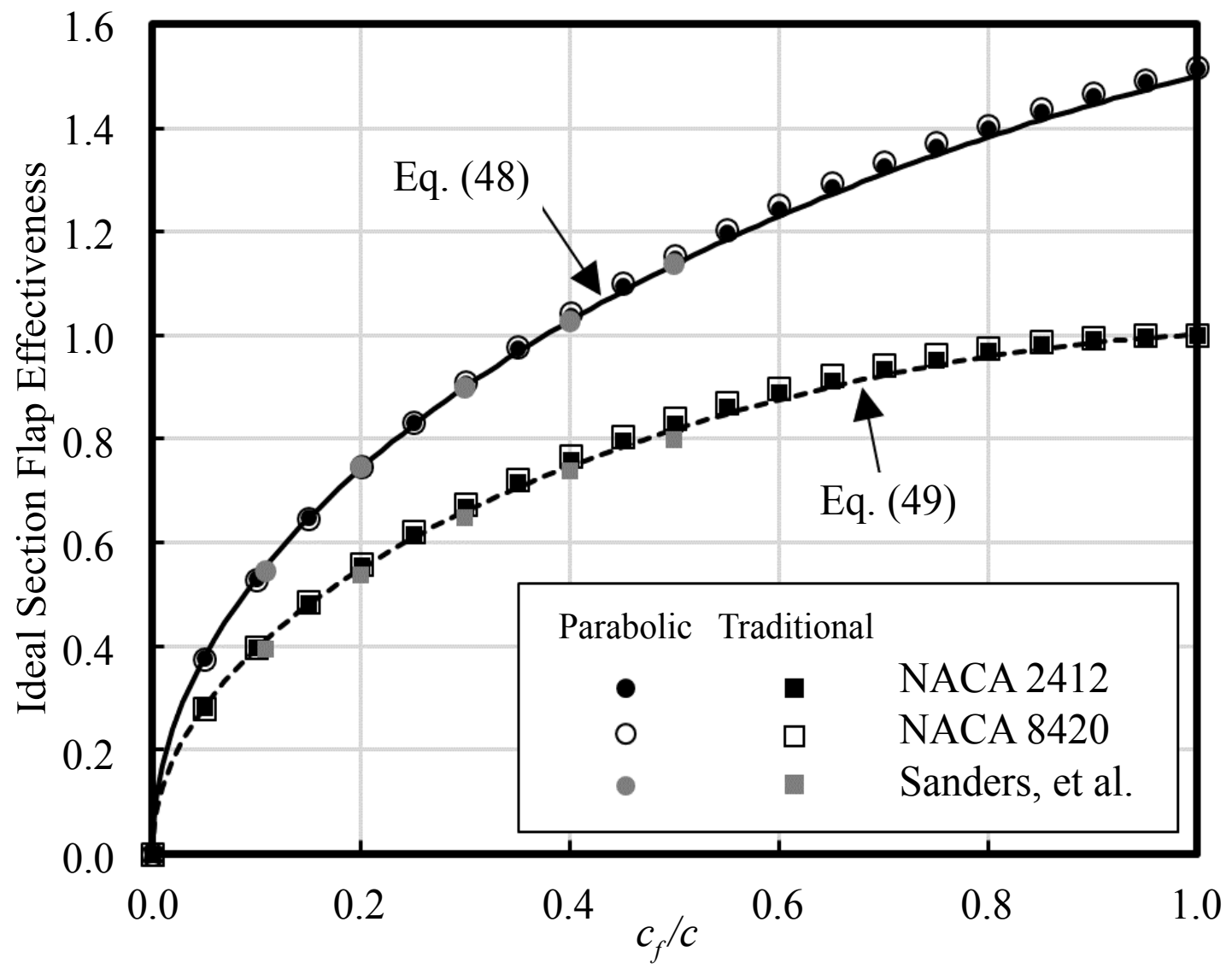

Figure 5: Ideal section flap effectiveness of traditional and parabolic flaps as predicted by thin airfoil theory, the vortex panel method, and Sanders, et al. [11]. 


$$
\alpha_{L 0}=\frac{1}{\pi} \int_{\theta=0}^{\pi} \frac{d y_{c 0}}{d x}(1-\cos \theta) d \theta-\frac{2 \delta_{p}}{\pi\left(1+\cos \theta_{f}\right)}\left[\int_{\theta=\theta_{f}}^{\pi}\left(\cos \theta_{f}-\cos \theta\right)(1-\cos \theta) d \theta\right]
$$

where

$$
\theta_{f} \equiv \cos ^{-1}\left(1-2 \frac{x_{f}}{c}\right)=\cos ^{-1}\left(2 \frac{c_{f}}{c}-1\right)
$$

is the flap hinge location, and the ratio $c_{f} / c$ is the flap-chord fraction. The first term in Eq. (46) is the zerolift angle of attack of the airfoil without any flap deflection. The change in zero-lift angle of attack for a given flap-chord fraction and flap deflection can be evaluated from the second term in Eq. (46). Note that within the small-angle and small-deflection approximations used, this term is directly proportional to the flap deflection, $\delta_{p}$. Integrating this term gives the ideal section flap effectiveness of a parabolic flap,

$$
\varepsilon_{i p} \equiv-\frac{\partial \alpha_{L 0}}{\partial \delta_{p}}=\frac{\left(1+2 \cos \theta_{f}\right)\left(\pi-\theta_{f}\right)+\sin \theta_{f}\left(2+\cos \theta_{f}\right)}{\pi\left(1+\cos \theta_{f}\right)}
$$

Following the same process, but using the camber-line slope given in Eq. (14) gives the ideal section flap effectiveness of a traditional flap [18,27,28]

$$
\varepsilon_{i f} \equiv-\frac{\partial \alpha_{L 0}}{\partial \delta_{f}}=\frac{1}{\pi} \int_{\theta=\theta_{f}}^{\pi}(1-\cos \theta) d \theta=1-\frac{\theta_{f}-\sin \theta_{f}}{\pi}
$$

From Eqs. (48) and (49) we see that the ideal section flap effectiveness of either flap geometry as predicted by thin airfoil theory depends on only the flap-chord fraction, and is independent of the flap deflection angle, airfoil camber-line distribution, or airfoil thickness distribution. Figure 5 shows the ideal section

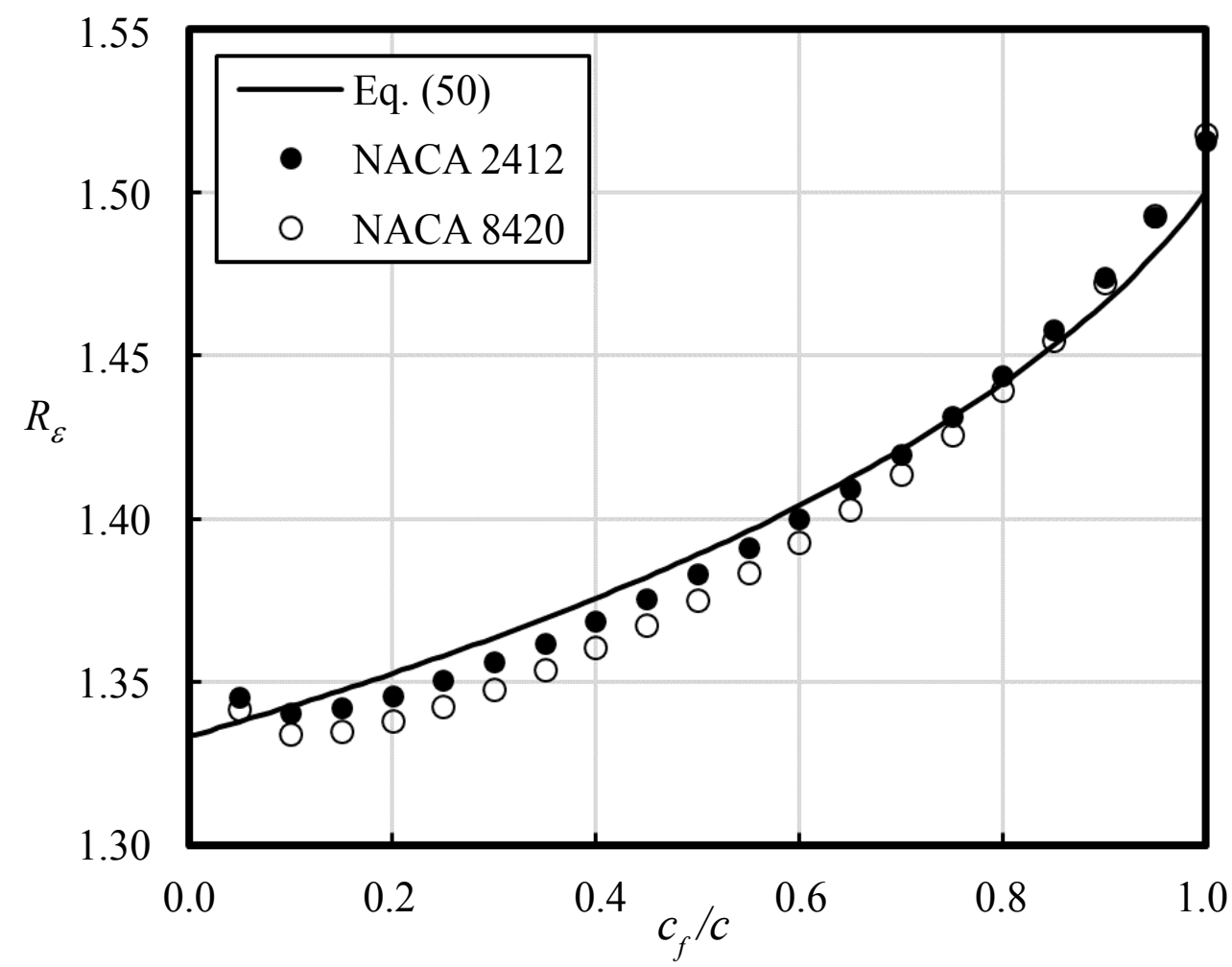

Figure 6: Parabolic-flap effectiveness ratio as predicted by thin airfoil theory and the vortex panel method. 
flap effectiveness of both traditional and parabolic flap geometries at small deflections as predicted by Eqs. (48) and (49). Results from a vortex panel method [29] for NACA 2412 and 8420 airfoils with each flap type are included for comparison. For the vortex-panel computations, 400 nodes around the airfoil surface were used to ensure grid convergence, and forward differencing with a step size of 1 deg deflection was used to compute the ideal section flap effectiveness. This data falls very near the analytical solutions given by Eqs. (48) and (49) and demonstrates that the ideal section flap effectiveness is only a weak function of camber and thickness when only potential flow is considered. Sanders, Eastep, and Forster [11] do not show plots of the ideal section flap effectiveness directly. However, they do include computational results for the change in lift coefficient per degree of flap deflection. These results were digitized and used to compute an estimate for the ideal section flap effectiveness from their work. These results are included in Figure 5 for comparison. Since their small-angle camber-line slope is the same as that given in Eq. (45), any deviation from the grey symbols and Eqs. (48) and (49) visible in Figure 5 are likely due to plot-digitization errors.

One measure of aerodynamic performance of the parabolic flap is the ratio of the ideal section flap effectiveness of the parabolic flap to that of the traditional flap. We will call this ratio the parabolic-flap effectiveness ratio. Dividing Eq. (48) by Eq. (49) gives the parabolic-flap effectiveness ratio as predicted from thin airfoil theory

$$
R_{\varepsilon} \equiv \frac{\varepsilon_{i p}}{\varepsilon_{i f}}=\frac{\left(1+2 \cos \theta_{f}\right)\left(\pi-\theta_{f}\right)+\sin \theta_{f}\left(2+\cos \theta_{f}\right)}{\left(1+\cos \theta_{f}\right)\left(\pi-\theta_{f}+\sin \theta_{f}\right)}
$$

Figure 6 shows the parabolic-flap effectiveness ratio as a function of flap-chord fraction computed from Eq. (50). Results are also included for the NACA 2412 and 8420 airfoils as computed from the vortex

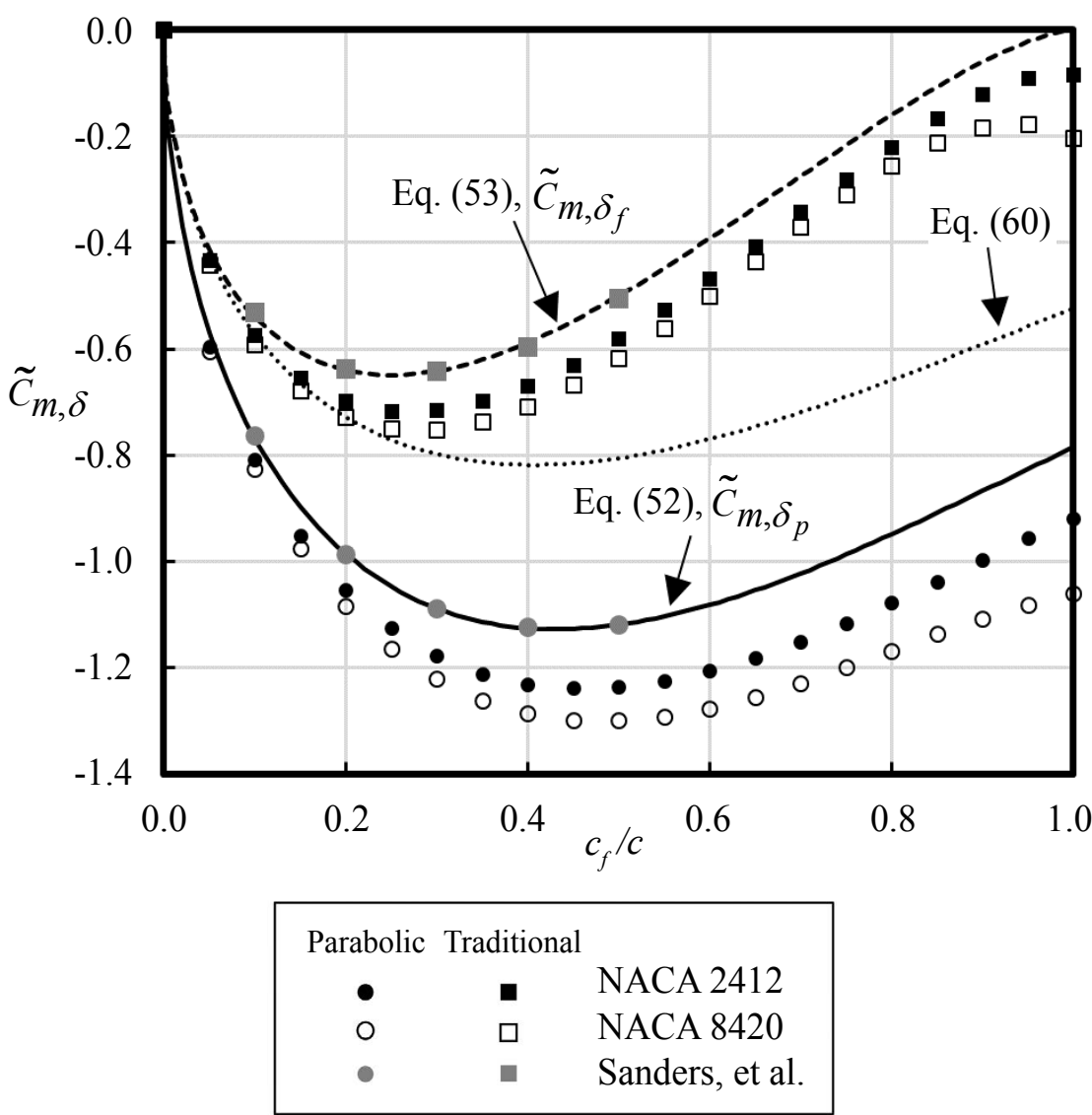

Figure 7: Change in quarter-chord pitching moment with respect to flap deflection for traditional and parabolic flaps as predicted by thin airfoil theory, the vortex panel method, and Sanders, et al. [11]. 
panel method. From Figure 5 and Figure 6 we see that a single degree of deflection from the parabolic flap produces significantly more lift than a single degree of deflection from a traditional flap. This is because a single degree of deflection of a parabolic flap produces a larger change in camber-line slope near the trailing edge than does a traditional flap. Hence, a parabolic flap will produce a larger change in lift than a traditional flap for a given flap-deflection angle. This should not be understood to mean that the parabolic flap is always more aerodynamically efficient than a traditional flap. Indeed, additional aerodynamic characteristics are also important, including the effect of the flap on airfoil pitching moment as well as the effects of viscosity and parasitic drag. However, within the limits of potential flow, the parabolic flap has an ideal section flap effectiveness ranging from $33.3 \%$ to $50 \%$ higher than that of a traditional flap. Numerical results from the NACA 2412 and 8420 airfoils show that camber and thickness produce results that deviate only slightly from the thin-airfoil approximation given in Eq. (50).

\section{Section quarter-chord pitching moment}

In a similar manner, we can use thin airfoil theory to estimate the change in section quarter-chord pitching moment due to flap deflection. Using Eq. (45) in Eq. (41) with the change of variables given in Eq. (44) gives

$$
\tilde{C}_{m_{c / 4}}=\frac{1}{2} \int_{\theta=0}^{\pi} \frac{d y_{c 0}}{d x}[\cos (2 \theta)-\cos \theta] d \theta-\frac{\delta_{p}}{1+\cos \theta_{f}} \int_{\theta=\theta_{f}}^{\pi}\left(\cos \theta_{f}-\cos \theta\right)[\cos (2 \theta)-\cos \theta] d \theta
$$

Within the approximations used for thin airfoil theory, the first term in Eq. (51) is exactly the section pitching moment of the airfoil with zero flap deflection, and the second term is proportional to the flap deflection. The change in section pitching moment with respect to flap deflection can be evaluated by integrating the second term and differentiating the result with respect to flap deflection,

$$
\tilde{C}_{m, \delta_{p}} \equiv \frac{\partial \tilde{C}_{m_{c / 4}}}{\partial \delta_{p}}=-\frac{3\left(\pi-\theta_{f}\right)+3 \sin \theta_{f}\left(2 \cos \theta_{f}+1\right)\left(1-\cos \theta_{f}\right)+\sin \left(3 \theta_{f}\right)}{6\left(1+\cos \theta_{f}\right)}
$$

Following the same process, but using the camber-line slope given in Eq. (14) gives the change in section pitching moment with respect to flap deflection of a traditional flap [28]

$$
\tilde{C}_{m, \delta_{f}} \equiv \frac{\partial \tilde{C}_{m_{c / 4}}}{\partial \delta_{f}}=-\frac{1}{2} \int_{\theta=\theta_{f}}^{\pi}[\cos (2 \theta)-\cos \theta] d \theta=\frac{\sin \left(2 \theta_{f}\right)-2 \sin \theta_{f}}{4}
$$

From Eqs. (52) and (53) we see that the change in section pitching moment with respect to flap deflection of either flap geometry is predicted by thin airfoil theory to depend on only the flap-chord fraction, and is independent of the flap deflection angle, original airfoil camber line distribution, or airfoil thickness distribution. Figure 7 shows the change in section quarter-chord pitching moment with respect to flap deflection for both traditional and parabolic flap geometries at small deflections as predicted by Eqs. (52) and (53). Results from a vortex panel method [29] for NACA 2412 and 8420 airfoils at zero degrees angle of attack with each flap type are included. The same node count and finite-differencing techniques as mentioned previously were used for these computations. Additionally, estimated results from linear potential aerodynamic computations published by Sanders, Eastep, and Forster [11] are included for comparison. Airfoil thickness tends to increase the magnitude of the change in pitching moment with respect to flap deflection, while viscosity and hinge effects can significantly decrease this magnitude [28]. Therefore, thin airfoil theory or vortex-panel results should only be used for preliminary design.

Notice that the change in section pitching moment with respect to flap deflection predicted by thin airfoil theory for the traditional flap goes to zero as the flap-chord fraction approaches 1 . This is because for a flap-chord fraction of 1 , a traditional flap deflection is equivalent to a rotation of the complete airfoil, and 


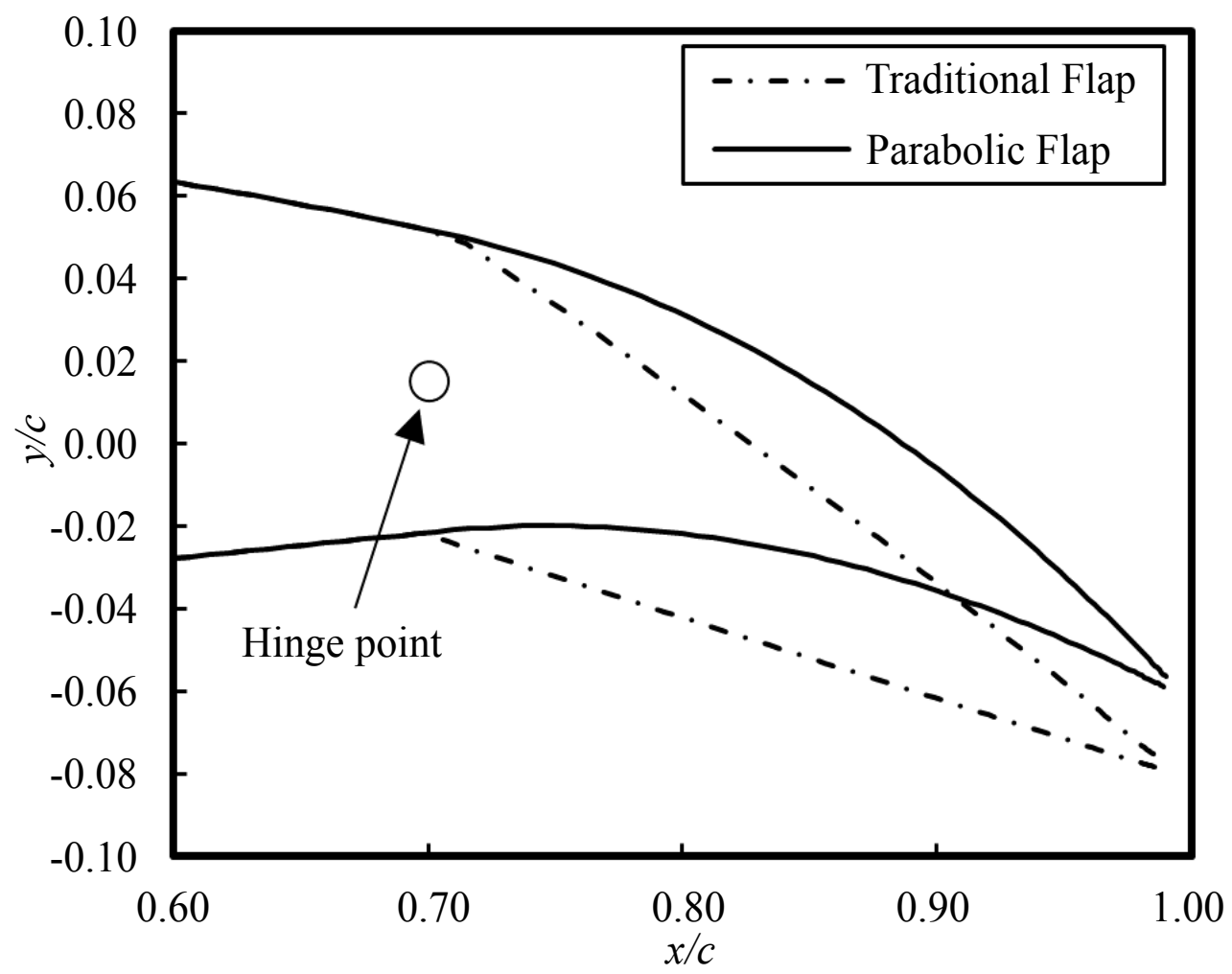

Figure 8: Example equivalent-lift deflection for the traditional flap (15.0 deg) and parabolic flap (11.23 deg) using a NACA 2412 airfoil with $c_{f} / c=0.3$, computed using a vortex panel method.

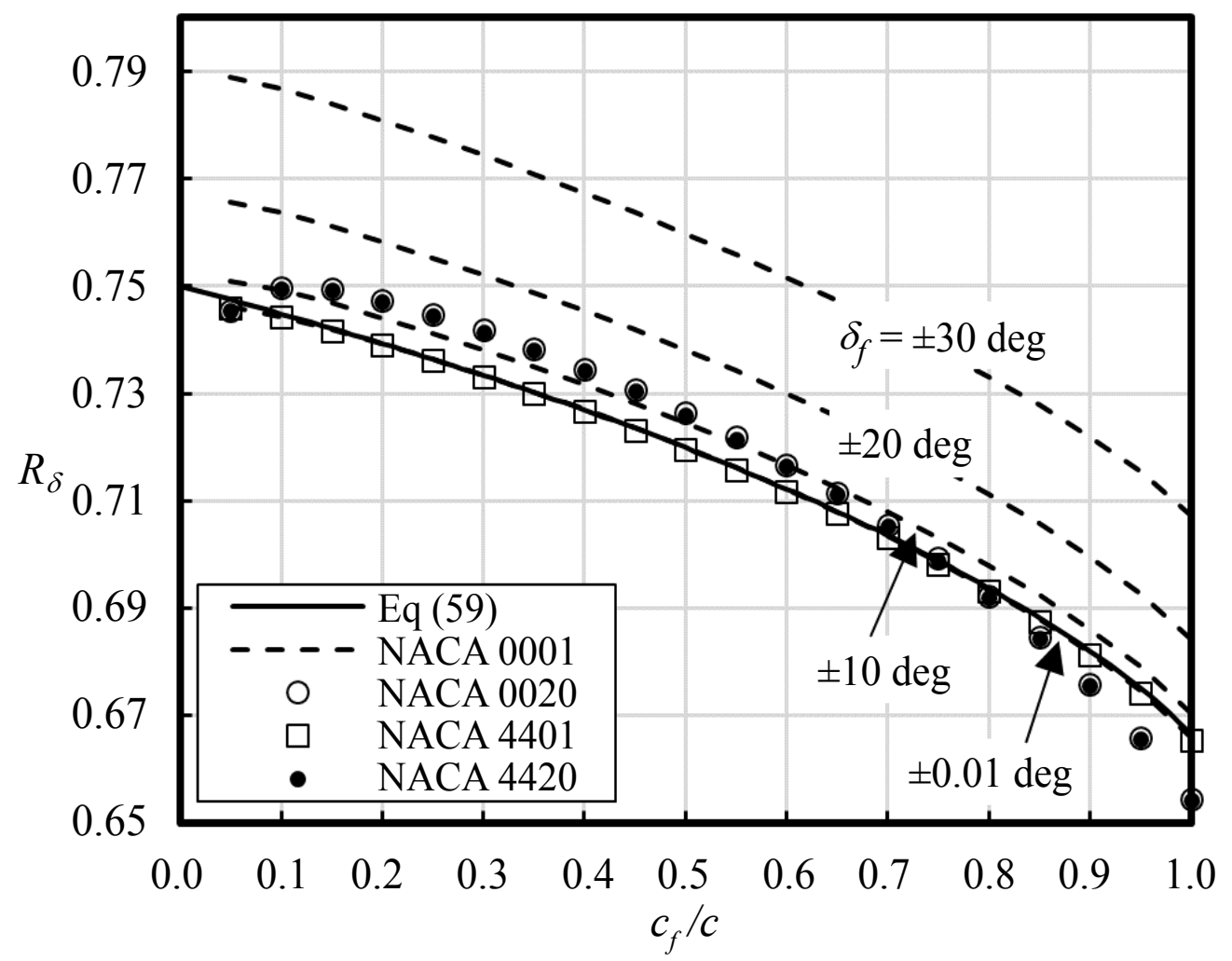

Figure 9: Equivalent-lift deflection ratio as a function of flap-chord fraction predicted from thin airfoil theory and the vortex panel method. 
therefore equivalent to a change in angle of attack. Since the aerodynamic center of an airfoil is predicted by thin airfoil theory to be located at the quarter chord, this theory also predicts zero change in section pitching moment about the quarter chord due to a change in flap deflection for $c_{f} / c=1$. On the other hand, a parabolic flap deflection for $c_{f} / c=1$ is not equivalent to a rotation of the complete airfoil. Rather, a parabolic flap deflection for this case has zero deflection at the leading edge, and a continuously increasing deflection along the chord, with the maximum deflection occurring at the trailing edge. Hence, for a parabolic flap, the change in section quarter-chord pitching moment with respect to flap deflection is nonzero for $c_{f} / c=1$. It is also interesting to note that the maximum absolute change in section pitching moment due to flap deflection for a traditional flap occurs in the range $0.2 \leq c_{f} / c \leq 0.3$, whereas that for the parabolic flap occurs in the range $0.4 \leq c_{f} / c \leq 0.6$.

\section{Equivalent-lift deflections}

In order to evaluate the aerodynamic performance of the two flap types, it is perhaps best to compare their performance at deflections that produce equivalent lift, i.e.,

$\tilde{C}_{L}\left(\delta_{p}\right)=\tilde{C}_{L}\left(\delta_{f}\right)$

Here we define an equivalent-lift deflection ratio, $R_{\delta}$, as the ratio of parabolic-flap deflection to traditional flap deflection required to produce the same amount of lift for a given flap-chord fraction and angle of attack, i.e.,

$$
\left.R_{\delta} \equiv \frac{\delta_{p}}{\delta_{f}}\right|_{\widetilde{C}_{L}, c_{f} / c, \alpha}
$$

For example, the equivalent-lift deflection ratio for a NACA 2412 airfoil with $c_{f} / c=0.3$ at an angle of attack of zero was computed using a vortex panel method. A traditional flap deflection of 15 deg was specified, and Newton's method was used to compute the parabolic flap deflection that would produce the same lift coefficient for the airfoil at the same flap-chord fraction and angle of attack. The equivalent parabolic-flap deflection was found to be
$11.23 \mathrm{deg}$, which gives an equivalent-lift deflection ratio of 0.748 . In this example, using a parabolic flap requires only about $75 \%$ of the deflection that would be required by a traditional flap to create the same change in lift coefficient. Figure 8 shows these equivalent-lift deflection geometries.

An estimate for the equivalent-lift deflection ratio for small deflections as a function of hinge location can be obtained from thin airfoil theory. Within the small-angle approximations of this theory, the lift coefficient is a linear function of the flap deflection for both traditional and parabolic flaps. For a parabolic flap, using Eqs. (46) and (48) in Eq. (40) gives the thin-airfoil-theory approximation for the lift coefficient at small flap deflections

$$
\tilde{C}_{L}=\tilde{C}_{L, \alpha}\left[\alpha-\alpha_{L 0}(0)+\varepsilon_{i p} \delta_{p}\right]
$$

where $\alpha_{L 0}(0)$ is the zero-lift angle of attack of the airfoil with no flap deflection. For a traditional flap, the lift coefficient at small flap deflections can likewise be written as

$$
\tilde{C}_{L}=\tilde{C}_{L, \alpha}\left[\alpha-\alpha_{L 0}(0)+\varepsilon_{i f} \delta_{f}\right]
$$

From the lift relations in Eqs. (56) and (57), we see that the equivalent-lift constraint from Eq. (54) requires

$\varepsilon_{i p} \delta_{p}=\varepsilon_{i f} \delta_{f}$

Using Eqs. (48) and (49) in Eq. (58) and rearranging, the equivalent-lift deflection ratio predicted by thin airfoil theory for small flap deflections is

$$
R_{\delta}=\frac{\left(1+\cos \theta_{f}\right)\left(\pi-\theta_{f}+\sin \theta_{f}\right)}{\left(1+2 \cos \theta_{f}\right)\left(\pi-\theta_{f}\right)+\sin \theta_{f}\left(2+\cos \theta_{f}\right)}=\frac{1}{R_{\varepsilon}}
$$

Figure 9 shows the equivalent-lift deflection ratio as a function of flap-chord fraction given by Eq. (59). Because this relation was developed using the small-angle approximation for flap deflection inherent from thin airfoil theory, we would not expect it to be accurate for large deflections. To demonstrate sample effects of deflection magnitude, results from the vortex panel method [29] are included for the NACA 0001 airfoil over a range of traditional flap deflection angles, $\delta_{f}$. Inviscid results from vortex panel methods may vary slightly from those shown 
in Figure 9 due to treatment of intersecting surfaces at large deflections. To demonstrate the effects of thickness and camber, results from the vortex panel method are included for NACA 0020, 4401, and 4420 airfoils for small deflections. Note that for small deflections, the equivalent-lift deflection ratio approaches 3/4 for small flap-chord fractions, and 2/3 for large flap-chord fractions. Airfoil thickness tends to increase this ratio for flap-chord fractions less than about 0.75 , and decrease this ratio for flap-chord fractions greater than 0.75 . Camber appears to have nearly negligible effect. The equivalent-lift deflection ratio increases for increasing deflection-angle magnitudes, and approaches $R_{\delta}=1$ as $\delta_{f} \rightarrow \pm 90 \mathrm{deg}$.

The changes in section quarter-chord pitching moment given by Eqs. (52) and (53) for the traditional-flap and parabolic-flap geometries were obtained by differentiating the section quarter-chord pitching moment with respect to flap deflection for each flap type. However, it is perhaps more insightful to consider the change in section quarter-chord pitching moment with respect to flap deflection for an equivalent change in lift produced by each flap type.

The ratio given in Eq. (59) provides an estimate for the flap-deflection magnitude required for a parabolic flap to create the same change in lift as a single degree of deflection of a traditional flap with the same flapchord fraction and angle of attack. Therefore, multiplying Eq. (52) by Eq. (59) gives the change in section quarter-chord pitching moment for a parabolic flap with respect to the flap deflection that produces the same change in lift as a traditional flap, as estimated by thin airfoil theory. This gives

$$
\left.\tilde{C}_{m, \delta_{p}}\right|_{\tilde{C}_{L}, c_{f} / c, \alpha}=-\frac{\left[3\left(\pi-\theta_{f}\right)+3 \sin \theta_{f}\left(2 \cos \theta_{f}+1\right)\left(1-\cos \theta_{f}\right)+\sin \left(3 \theta_{f}\right)\right]\left(\pi-\theta_{f}+\sin \theta_{f}\right)}{6\left[\left(1+2 \cos \theta_{f}\right)\left(\pi-\theta_{f}\right)+\sin \theta_{f}\left(2+\cos \theta_{f}\right)\right]}
$$

Predictions from Eq. (60) are included in Figure 7. Note that the parabolic flap generates a larger change in section pitching moment than does the traditional flap for an equivalent change in lift.

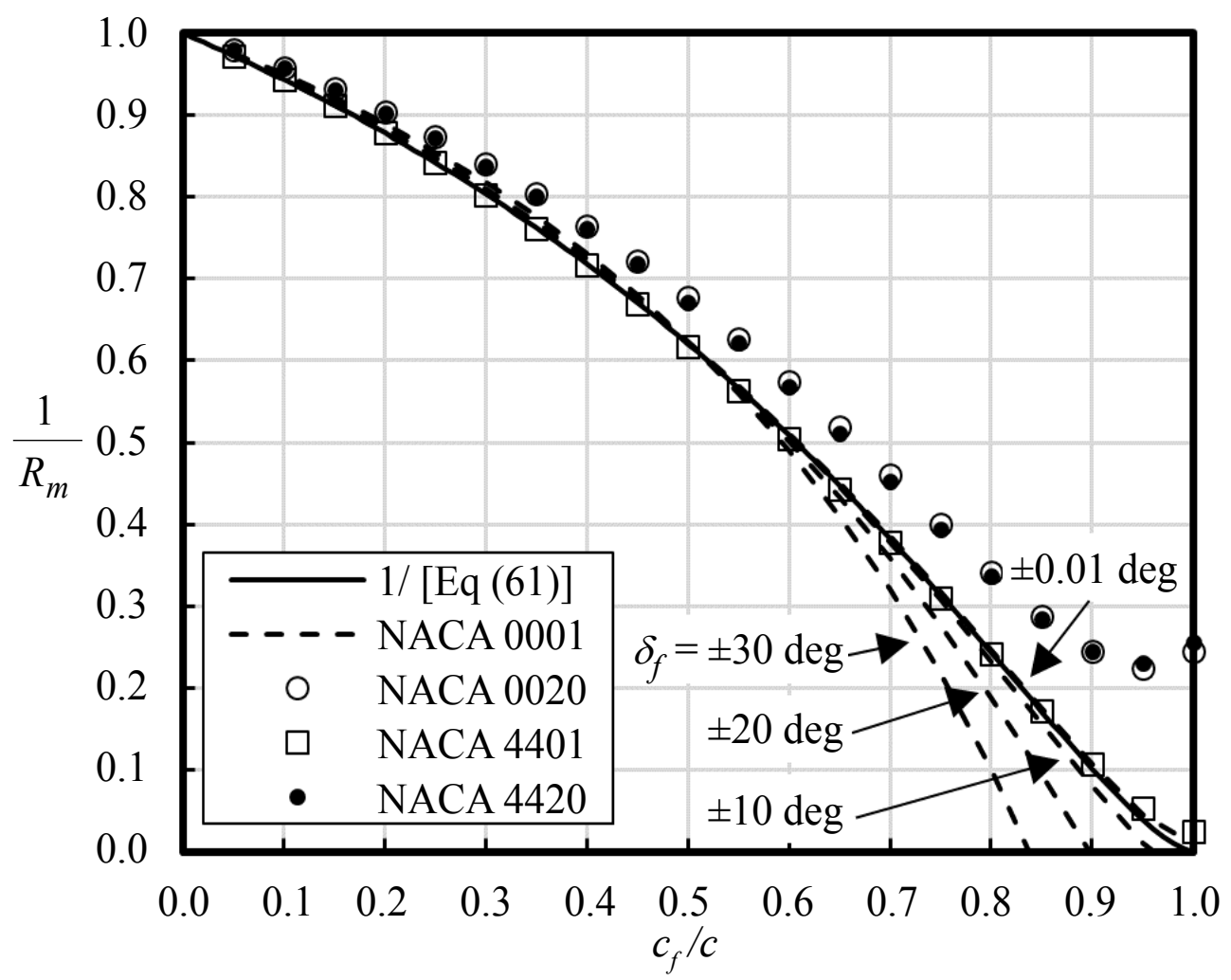

Figure 10: Equivalent-lift pitching-moment ratio as a function of flap-chord fraction predicted from thin airfoil theory and the vortex panel method. 
The ratio of the section pitching moment produced by the parabolic flap to that produced by the traditional flap for an equivalent change in lift will be termed the equivalent-lift pitching-moment ratio. An estimate for this ratio can be obtained from thin airfoil theory by dividing Eq. (60) by Eq. (53), which gives

$$
\left.R_{m} \equiv \frac{\tilde{C}_{m, \delta_{p}}}{\tilde{C}_{m, \delta_{f}}}\right|_{\tilde{C}_{L}, c_{f} / c, \alpha}=-\frac{2\left[3\left(\pi-\theta_{f}\right)+3 \sin \theta_{f}\left(2 \cos \theta_{f}+1\right)\left(1-\cos \theta_{f}\right)+\sin \left(3 \theta_{f}\right)\right]\left(\pi-\theta_{f}+\sin \theta_{f}\right)}{3\left[\left(1+2 \cos \theta_{f}\right)\left(\pi-\theta_{f}\right)+\sin \theta_{f}\left(2+\cos \theta_{f}\right)\right]\left[\sin \left(2 \theta_{f}\right)-2 \sin \theta_{f}\right]}
$$

Because Eq. (53) approaches zero as the flap-chord fraction approaches unity, the ratio in Eq. (61) approaches infinity as the flap-chord fraction becomes large. Therefore, to observe the trend suggested by Eq. (61), the inverse of Eq. (61) (i.e. $1 / R_{m}=\widetilde{C}_{m, \delta_{f}} / \widetilde{C}_{m, \delta_{p}}$ ) is plotted as a function of flap-chord fraction in Figure 10. Again, to demonstrate the effects of deflection, results are included for the NACA 0001 airfoil with varying deflection magnitudes. To demonstrate the effects of thickness and camber, results are included for the NACA 0020, 4401, and 4420 airfoils with small deflections. Typical flap-chord fractions of traditional flaps generally range between 0.1 and 0.4 . In this range, the traditional flap creates only $70-95 \%$ the magnitude of pitching moment created by the parabolic flap for the equivalent-lift deflection. As is true for aircraft employing traditional flaps, the change in pitching moment as a result of flap deflection can be significant and should be accounted for during the design process of any aircraft employing parabolic flaps. However, because viscosity can have a significant impact on the pitching moment, the results presented here based on ideal aerodynamics should be used with caution.

\section{Conclusions}

The geometry of a parabolic flap has been defined here as that produced by a parabolic deflection of the flap neutral line aft of a specified hinge point. This geometry can be generated for any arbitrary airfoil using the methodology outlined in this paper, provided that the camber line and thickness distributions of the airfoil are known. The methodology requires a numerical solver to ensure that the length of the flap neutral line does not change with deflection. The resulting parabolic-flap geometry has a camber-line slope that is continuous across the hinge point, whereas the camber-line slope of the traditional flap has a discontinuity across the hinge point.

Thin airfoil theory has been used to find analytical solutions for the ideal section flap effectiveness and change in section quarter-chord pitching moment with respect to flap deflection of the parabolic flap. These analytical solutions are given in Eqs. (48) and (52) respectively, and shown in Figure 5 and Figure 7 respectively, in comparison to thin-airfoil-theory results for the traditional flap. Solutions from inviscid computations using a vortex panel method are included to demonstrate the effects of thickness and camber. Results show that the ideal section flap effectiveness of a parabolic flap can range from $33 \%$ to $50 \%$ greater than that of a traditional flap, depending on the flap-chord fraction, with larger flap-chord fractions producing the largest gains in ideal section flap effectiveness. It was found that thickness and camber can have a significant effect on the change in section pitching moment due to flap deflection, but only a small effect on the ideal section flap effectiveness.

Estimates for the parabolic-flap effectiveness ratio, equivalent-lift deflection ratio, and equivalent-lift pitching-moment ratio were obtained from thin airfoil theory and given in Eqs. (50), (59), and (61) respectively. Results are shown in Figure 6, Figure 9, and Figure 10 in comparison to vortex panel solutions for NACA airfoils demonstrating the effects of camber and thickness. These results show that the parabolic flap typically requires $65-80 \%$ of the deflection of a traditional flap to produce the same change in lift, depending on flap-chord fraction and deflection magnitude. Additionally, within the range of traditional flap-chord fractions, the parabolic flap can create a change in pitching moment that is $5 \%$ to $50 \%$ larger than that of a traditional flap for the same change in lift, with the largest differences in pitching moment occurring at 
larger flap-chord fractions. The vortex-panel solutions demonstrate that camber and thickness appear to have only a small effect on the equivalent-lift deflection ratio, but that the deflection magnitude can have a significant effect on this ratio.

The present study used only ideal aerodynamics to evaluate the aerodynamic performance of a parabolic flap to that of a traditional flap. Ideal-aerodynamic estimates neglect viscosity, and therefore do not provide insight into either the lift-to-drag ratio, or the viscous results of adverse-pressure gradients on the airfoil surface. Future work is planned to understand these effects through the use of windtunnel measurements, computational fluid dynamics, and boundary-layer theory. Although these future studies will shed significantly more light on the true aerodynamic performance of a parabolic flap in comparison to a traditional flap, the results in this paper provide the analytical foundation with which these flap types can be compared.

\section{Acknowledgements}

This work was partially funded by the Air Force Office of Scientific Research (AFOSR) Lab Task 17RQCOR394 with Dr. Jay Tiley as the program manager and the AFRL Summer Faculty Fellowship Program. This paper has been cleared for public release, Case Number: 88ABW-2018-1444.

\section{References}

1. Joo J, Marks C, Zientarski L, Culler A (2015) Variable camber compliant wing-Design. AIAA-2015-1050, $23^{\text {rd }}$ AIAA/AHS Adaptive Structures Conference, Kissimmee, Florida.

2. Marks CR, Zientarski L, Culler AJ, Hagen B, Smyers $\mathrm{BM}$, et al. (2015) Variable camber compliant wingWind Tunnel Testing. AIAA 2015-1051, $23^{\text {rd }}$ AIAA/ AHS Adaptive Structures Conference, Kissimmee, Florida.

3. Nguyen N, Lebofsky S, Ting E, Kaul U, Chaparro D, et al. (2015) Development of variable camber continuous trailing edge flap for performance of adaptive aeroelastic wing. SAE Technical Paper.

4. Kaul UK, Nguyen NT (2014) Drag optimization study of Variable Camber Continuous Trailing Edge Flap (VCCTEF) Using OVERFLOW. $32^{\text {nd }}$ AIAA Applied Aerodynamic Conference, Aviation, Georgia.

5. Abdullah EJ, Bil C, Watkins S (2010) Numerical simulation of an adaptive airfoil system using SMA
Actuators. $48^{\text {th }}$ AIAA Aerospace Sciences Meeting, Florida.

6. Woods BKS, Friswell MI (2012) Preliminary investigation of a fishbone active camber concept. Proceedings of the ASME 2012 Conference on Smart Materials, Adaptive Structures and Intelligent Systems, Stone Mountain, Georgia.

7. Woods BKS, Bilgen O, Friswell MI (2012) Wind tunnel testing of the fish bone active camber morphing concept. $23^{\text {rd }}$ International Conference on Adaptive Structures and Technologies, China.

8. Vasista S, Tong L (2013) Topology-optimized design and testing of a pressure-driven morphing-aerofoil trailing-edge structure. AIAA Journal 51: 1898-1907.

9. Lyu Z, Martins JRRA (2015) Aerodynamic shape optimization of an adaptive morphing trailing-edge wing. Journal of Aircraft 52: 1951-1970.

10.Liu Y, Bai J, Livne E (2017) Robust optimization of variable-camber continuous trailing-edge flap static aeroelastic action. AIAA Journal 55: 1031-1043.

11. Sanders B, Eastep FE, Forster E (2003) Aerodynamic and aeroelastic characteristics of wings with conformal control surfaces for morphing aircraft. Journal of Aircraft 40: 94-99.

12.Pankonien AM (2015) Smart material wing morphing for unmanned aerial vehicles. PhD Dissertation, University of Michigan.

13.Abbott IH, Von Doenhoff AE (1959) Theory of wing sections. Dover, New York, 111-123.

14.Munk MM (1922) General theory of thin wing sections. NACA TR-142.

15.Birnbaum W (1923) Die tragende Wirbelfläche als Hilfsmittel zur Behandlung des ebenen Problems der Tragflügeltheorie. Zeitschrift für Angewandte Mathematik und Mechanik 3: 290-297.

16. Glauert H (1924) A theory of thin aerofoils. Aeronautical Research Council, Reports and Memoranda 910, London.

17. Glauert H (1926) Thin aerofoils. The Elements of Aerofoil and Airscrew Theory, Cambridge Univ Press, UK, 87-93.

18. Glauert H (1927) Theoretical relationships for an aerofoil with hinged flap. Aeronautical Research Council, Reports and Memoranda 1095, London.

19.Abbott IH, Von Doenhoff AE (1959) Theory of Thin Wing Sections. Theory of Wing Sections, McGrawHill, New York, 64-79.

20.Karamcheti K (1966) Elements of thin airfoil theory. Principles of Ideal-Fluid Aerodynamics, Krieger, Malabar, FL, 492-517. 
21.Anderson JD (2011) Classical thin airfoil theory. Fundamentals of Aerodynamics. ( $5^{\text {th }}$ edn), McGrawHill, New York, 338-357.

22.Bertin JJ, Cummings RM (2014) Thin-airfoil theory. Aerodynamics for Engineers. ( $6^{\text {th }}$ edn $)$, Prentice-Hall, Upper Saddle River, NJ, 298-317.

23.Katz J, Plotkin A (2001) Small-Disturbance flow over two-dimensional airfoils. Low-Speed Aerodynamics. ( $2^{\text {nd }}$ edn), Cambridge Univ Press, UK, 94-121.

24.Kuethe AM, Chow CY (1998) Aerodynamic characteristics of airfoils. Foundations of Aerodynamics: Bases of Aerodynamic Design. ( $5^{\text {th }}$ edn), Wiley, New York, 136-150.

25.McCormick BW (1995) Thin airfoil theory. Aerody- namics, Aeronautics, and Flight Mechanics. ( $2^{\text {nd }}$ edn $)$, Wiley, New York, 73-84.

26.Phillips WF (2010) Thin airfoil theory. Mechanics of Flight. ( $\left.2^{\text {nd }} e d n\right)$, Wiley, Hoboken, NJ, 28-32.

27. Abbott IH, Von Doenhoff AE (1959) Plain Flaps. Theory of Wing Sections, McGraw-Hill, New York, 190-197.

28.Phillips WF (2010) Trailing-edge flaps and section flap effectiveness. Mechanics of Flight. ( $2^{\text {nd }}$ edn), Wiley, Hoboken, NJ, 39-46.

29.Phillips WF (2010) The vortex panel method. Mechanics of Flight. ( $\left.2^{\text {nd }} e d n\right)$, Wiley, Hoboken, NJ, 32-39. 